M. YASUDA

KODAI MATH. J.

31 (2008), 385-403

\title{
TORSION POINTS OF ELLIPTIC CURVES WITH GOOD REDUCTION
}

\author{
Masaya Yasuda
}

\begin{abstract}
We consider the torsion points of elliptc curves over certain number fields with good reduction everywhere. ${ }^{1}$
\end{abstract}

\section{Introduction}

It is well known that there do not exist elliptic curves over $\mathbf{Q}$ with good reduction everywhere. The existence of elliptic curves with good reduction everywhere over quadratic fields was observed by Comalada [2]. We recall that an admissible elliptic curve over a number field $K$ is an elliptic curve defined over $K$, which has good reduction everywhere with a non-trivial 2-division point rational over $K$. Comalada classified admissible elliptic curves over real quadratic fields, dealing with certain diophantine equations in units of real quadratic fields (see [2]). In his paper [7], Kida computed the torsion subgroup of the Mordell-Weil group of admissible elliptic curves over certain real quadratic fields and showed that an admissible elliptic curve over a certain quadratic field $K$ has only $K$-rational points of order $p$ for small prime $p$. In this paper, we consider the torsion points of prime order of elliptic curves over certain number fields with good reduction everywhere. For each prime number $p$, we let $\zeta_{p}$ denote a primitive $p$-th root of unity. Our main result is the following:

THEOREM 0.1. Let $K$ be a number field having a real place and let $p$ be a prime number. Suppose that $p$ does not divide the class number of $K\left(\zeta_{p}\right)$ and the ramification index $e_{\mathfrak{p}}$ satisfies $e_{\mathfrak{p}}<p-1$ for all primes $\mathfrak{p}$ of $K$ above $p$. Let $E$ be an elliptic curve over $K$ with good reduction everywhere. Then $E$ has no $K$-rational points of order $p$.

Let $p$ be an odd prime number. Let $K$ be a number field and let $\mathcal{O}_{K}$ denote its ring of integers. Our main idea to prove above result is to examine the extensions of a diagonalizable group scheme $\mu_{p}$ by a constant group scheme $\mathbf{Z} / p \mathbf{Z}$ over the ring $\mathcal{O}_{K}$. Schoof studied the extensions of $\mu_{p}$ by $\mathbf{Z} / p \mathbf{Z}$ over $\mathcal{O}_{K}$,

${ }^{1} 2000$ Mathematics Subject Classification. Primary 14L15; Secondary 11G05.

Received August 29, 2007; revised February 29, 2008. 
using the equivalence of categories between the category of $\mathcal{O}_{K}$-group schemes and the category of triples $\left(G_{1}, G_{2}, \theta\right)$ where $G_{1}$ is a finite flat $\hat{\mathcal{O}}_{K}$-group scheme, $G_{2}$ is a finite flat $\mathcal{O}_{K}[1 / p]$-group scheme and $\theta: G_{1} \otimes \hat{\mathcal{O}}_{K}[1 / p] \rightarrow G_{2} \otimes \hat{\mathcal{O}}_{K}[1 / p]$ is an isomorphism of $\hat{\mathcal{O}}_{K}[1 / p]$-group schemes (see [9]). Here the ring $\hat{\mathcal{O}}_{K}$ is the inverse limit of the ring $\mathcal{O}_{K} / p^{n} \mathcal{O}_{K}$ for $n \in \mathbf{N}$. In a similar way, we consider the extensions of $\mathbf{Z} / p \mathbf{Z}$ by $\mu_{p}$ over $\mathcal{O}_{K}$. In order to study the extensions of $\mathbf{Z} / p \mathbf{Z}$ by $\mu_{p}$ over $\mathcal{O}_{K}$, we calculate the extensions of $\mathbf{Z} / p \mathbf{Z}$ by $\mu_{p}$ over the completion $\mathcal{O}_{K, \boldsymbol{p}}$ at the prime $\mathfrak{p}$ of $K$ over $p$ by using Dieudonné theory (see [3]).

Finally we study the finite flat group schemes of prime order over the ring of integers of imaginary quadratic fields $K$ with class number one. In applications, we consider the existence of abelian varieties over $K$ with good reduction everywhere. The existence of such abelian varieties over cyclotomic fields was studied by Schoof (see [9]). According to Schoof's result, there do not exist non-zero abelian varieties over $K=\mathbf{Q}(\sqrt{m})$ with good reduction everywhere for $m \in\{-1,-2,-3,-7,-11\}$ under the Generalized Riemann Hypothesis (see [9]). Using Schoof's approach for the non-existence results of abelian varieties with good reduction everywhere, we get the following result:

THEOREM 0.2. Let $K=\mathbf{Q}(\sqrt{m})$ be an imaginary quadratic field with class number one and let $p$ be an odd prime number such that $p$ does not divide $m$ and $(m / p)=1$. Suppose that $p$ does not divide the class number of $K\left(\zeta_{p}\right)$. Let $A$ be an abelian variety over $K$ with bad reduction only at the primes of $K$ over $p$. Then $A$ has no complex multiplication over $K$.

As a corollary, we get the following result:

COROllary 0.3. There do not exist non-zero abelian varieties over $K=$ $\mathbf{Q}(\sqrt{-19})$ with good reduction everywhere and complex multiplication over $K$.

Notation. The symbols $\mathbf{Z}, \mathbf{Q}$, and $\mathbf{C}$ denote, respectively, the ring of rational integers, the field of rational numbers, and the field of complex numbers. If $G$ is a group scheme over a ring $R$, and $n \in \mathbf{Z}$, we write $G[n]$ for the kernel of multiplication $[n]_{G}: G \rightarrow G$.

\section{Finite flat group schemes over complete discrete valuation rings with low ramification}

Let $A=W(k)$ be the ring of Witt vectors over a perfect field $k$ of characteristic $p>0$. Let $\sigma$ be the Frobenius automorphism on $k$ and $A$. We consider the Dieudonné ring $D_{k}=A[F, V]$, where $F V=V F=p$, and for each $\lambda \in A, F \lambda=\sigma(\lambda) F$ and $\lambda V=V \sigma(\lambda)$. Let $\left(A^{\prime}, \mathfrak{m}\right)$ be the valuation ring of a finite totally ramified extension $K^{\prime}$ of $K$, with $e=\left[K^{\prime}: K\right]$ the absolute ramification index of $A^{\prime}$. Assume $e<p-1$. The category of finite flat commutative group schemes over $A$ with $p$-power order is denoted by $\mathscr{F}_{A} \mathscr{F}_{A}$, and $\widetilde{\mathscr{F} \mathscr{F}}_{A}$ is the full subcategory of objects killed by $p$. We define $\widetilde{\mathscr{F}}_{A^{\prime}}, \widetilde{\mathscr{F}}_{\mathscr{F}_{A}}$, etc. 
in a similar manner. Using the anti-equivalence from $\widetilde{\mathscr{F} \mathscr{F}}_{A^{\prime}}$ to the category of finite Honda systems killed by $p$, we calculate the extensions of group schemes over $A^{\prime}$ of order $p$.

\subsection{Review of Honda systems}

We recall here the theory of Honda systems (cf. [3]).

For each finite $k$-algebra $R_{k}$ with radical $R_{k}^{0}$, we set

$$
C W_{k}\left(R_{k}\right)=\left\{\bar{f}=\left(f_{-n}\right)_{n \geq 0} \mid f_{-n} \in R_{k} \text { and for almost all } n, f_{-n} \in R_{k}^{0}\right\} .
$$

Let $S_{m} \in \mathbf{Z}\left[X_{0}, \ldots, X_{m} ; Y_{0}, \ldots, Y_{m}\right]$ denote the $m$-th addition polynomial for $p$ Witt vectors. The functor $C W_{k}$ is a group functor with respect to the operation

$$
\left(f_{-n}\right)_{n \geq 0}+\left(g_{-n}\right)_{n \geq 0}=\left(h_{-n}\right)_{n \geq 0}
$$

where

$$
h_{-n}=\lim _{m \rightarrow \infty} S_{m}\left(f_{-n-m}, \ldots, f_{-n} ; g_{-n-m}, \ldots, g_{-n}\right) .
$$

The structure of $D_{k}$-module on $C W_{k}$ is defined by the relations

$$
\begin{aligned}
F\left(\left(f_{-n}\right)_{n \geq 0}\right) & =\left(\ldots, f_{-n}^{p}, \ldots, f_{0}^{p}\right), \\
V\left(\left(f_{-n}\right)_{n \geq 0}\right) & =\left(\ldots, f_{-n-1}, \ldots, f_{-1}\right), \\
{[\alpha]\left(\left(f_{-n}\right)_{n \geq 0}\right) } & =\left(\ldots,\left(\sigma^{-n} \alpha\right) f_{-n}, \ldots, \alpha f_{0}\right),
\end{aligned}
$$

where $\alpha \in k$ and $[\alpha]=(\ldots, 0,0, \alpha) \in W(k)=A$ is the Teichmüller representative for $\alpha$. Let $G_{k}=\operatorname{Spec} R_{k}$ be a $p$-group scheme over $k$ and let $\triangle: R_{k} \rightarrow R_{k} \otimes R_{k}$ be the comultiplication. For each $\bar{f}=\left(f_{-n}\right)_{n \geq 0} \in C W_{k}\left(R_{k}\right)$, we set $\triangle \bar{f}=$ $\left(\triangle f_{-n}\right)_{n \geq 0} \in C W_{k}\left(R_{k} \otimes R_{k}\right), \quad$ similarly, $\quad \bar{f} \otimes 1=\left(f_{-n} \otimes 1\right)_{n \geq 0} \quad$ and $\quad 1 \otimes \bar{f}=$ $\left(1 \otimes f_{-n}\right)_{n \geq 0}$. We set

$$
M\left(G_{k}\right)=\left\{\bar{f} \in C W_{k}\left(R_{k}\right) \mid \triangle \bar{f}=\bar{f} \otimes 1+1 \otimes \bar{f}\right\}=\operatorname{Hom}\left(G_{k}, C W_{k}\right),
$$

where the structure of $D_{k}$-module on $M\left(G_{k}\right)$ is induced by the corresponding structure on $C W_{k}$.

Let $M$ be a $D_{k}$-module. Define $M^{(1)}=A \otimes_{A} M$ as a $D_{k}$-module, using $\sigma: A \rightarrow A$, with operators $F(\lambda \otimes x)=\sigma(\lambda) \otimes F(x)$ and $V(\lambda \otimes x)=$ $\sigma^{-1}(\lambda) \otimes V(x)$. We have $A$-linear maps $F_{0}: M^{(1)} \rightarrow M$ and $V_{0}: M \rightarrow M^{(1)}$, with $F_{0} V_{0}=p_{M}$ and $V_{0} F_{0}=p_{M^{(1)}}$. We define $M_{A^{\prime}}$ to be the direct limit of the diagram

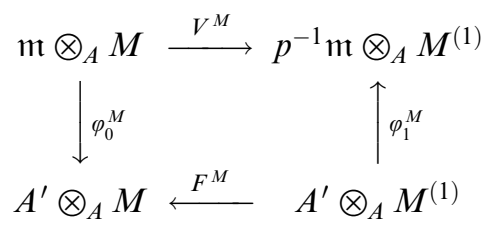

in the category of $A^{\prime}$-modules, where $\varphi_{0}^{M}, \varphi_{1}^{M}$ are the obvious maps, $V^{M}(\lambda \otimes x)=p^{-1} \lambda \otimes V_{0}(x)$, and $F^{M}(\lambda \otimes x)=\lambda \otimes F_{0}(x)$. More explicity, $M_{A^{\prime}}$ 
is the quotient of $\left(A^{\prime} \otimes_{A} M\right) \oplus\left(p^{-1} \mathfrak{m} \otimes_{A} M^{(1)}\right)$ by the submodule

$$
\left\{\left(\varphi_{0}^{M}(u)-F^{M}(w), \varphi_{1}^{M}(w)-V^{M}(u)\right) \mid u \in \mathfrak{m} \otimes_{A} M, w \in A^{\prime} \otimes_{A} M^{(1)}\right\} .
$$

There are canonical $A^{\prime}$-linear maps

$$
\begin{aligned}
& l_{M}: A^{\prime} \otimes_{A} M \rightarrow M_{A^{\prime}}, \\
& \mathscr{F}_{M}: p^{-1} \mathrm{~m} \otimes_{A} M^{(1)} \rightarrow M_{A^{\prime}}, \\
& \mathscr{V}_{M}: M_{A^{\prime}} \rightarrow A^{\prime} \otimes_{A} M^{(1)}
\end{aligned}
$$

(the last one induced by $1 \otimes V_{0}$ on $A^{\prime} \otimes_{A} M$ and $p \otimes$ id on $p^{-1} \mathfrak{m} \otimes_{A} M^{(1)}$ ). Using the natural $A$-linear maps $M \rightarrow A^{\prime} \otimes_{A} M \stackrel{l_{M}}{\rightarrow} M_{A^{\prime}}$ and $M^{(1)} \rightarrow p^{-1} \mathrm{~m} \otimes_{A} M^{(1)}$, we have the commutative diagram

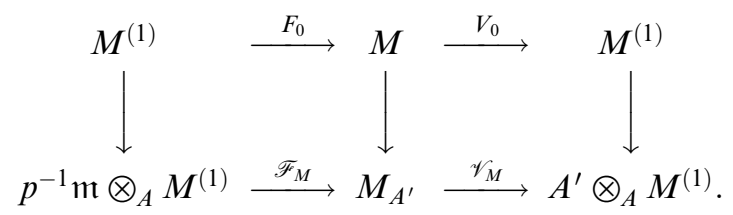

When $M$ has finite $A$-length, the commutative diagram above induces $k$-linear isomorphisms

$$
\begin{array}{ll}
\text { Ker } F_{0} \simeq \operatorname{Ker} \mathscr{F}_{M}, & \text { Coker } F_{0} \simeq \operatorname{Coker} \mathscr{F}_{M}, \\
\operatorname{Ker} V_{0} \simeq \operatorname{Ker} \mathscr{V}_{M}, & \text { Coker } V_{0} \simeq \operatorname{Coker} \mathscr{V}_{M}
\end{array}
$$

(see [3, Lemma 2.4]). The functor $M \leadsto M_{A^{\prime}}$ is exact on the category of $D_{k^{-}}$ modules of finite $A$-length (see [3, Lemma 2.2]).

Fix $G=\operatorname{Spec} R \in \mathscr{\mathscr { F }}_{A^{\prime}}$. We denote by $R_{k}$ and $R_{K^{\prime}}$ the closed and generic fibers respectively of $R$ over $A^{\prime}$. Set $M=M\left(G_{k}\right)$, where $G_{k}=\operatorname{Spec} R_{k} \in \mathscr{F}_{\mathscr{F}_{k}}$. Define a continuous $A$-linear map

$$
w_{R}: C W_{k}\left(R_{k}\right) \rightarrow R_{K^{\prime}} / p R
$$

by

$$
w_{R}\left(\left(a_{-n}\right)\right)=\sum_{n \geq 0} p^{-n} \hat{a}_{-n}^{p^{n}} \quad(\bmod p R),
$$

where $\hat{a}_{-n} \in R$ is a lift of $a_{-n} \in R_{k}$ (see [5, Ch. II, Section 5.2]). We define $L_{A^{\prime}}(G)$ to be the kernel of the $A^{\prime}$-linear map

$$
M_{A^{\prime}} \rightarrow C W_{k, A^{\prime}}\left(R_{k}\right)=\left(C W_{k}\left(R_{k}\right)\right)_{A^{\prime}} \stackrel{w_{R}^{\prime}}{\rightarrow} R_{K^{\prime}} / \mathrm{m} R
$$

where $w_{R}^{\prime}$ is induced by $w_{R}$ and a natural surjection $A^{\prime} \otimes_{A} C W_{k}\left(R_{k}\right) \rightarrow$ $C W_{k, A^{\prime}}\left(R_{k}\right)$. The objects of the category $S H_{A^{\prime}}^{f}$ of finite Honda systems over $A^{\prime}$ consist of $(L, M)$ where $M$ is a $D_{k}$-module of finite $A$-length and where $L$ is an $A^{\prime}$-submodule of $M_{A^{\prime}}$ such that the canonical $k$-linear map

$$
L / \mathrm{m} L \rightarrow \text { Coker } \mathscr{F}_{M}
$$


is an isomorphism and the restriction of $\mathscr{V}_{M}$ to $L \subseteq M_{A^{\prime}}$ is injective. The full subcategory of objects killed by $p$ is denoted by $\widetilde{S H}_{A^{\prime}}^{f}$. For any $G$ in $\mathscr{F} \mathscr{F}_{A^{\prime}}$, we define $L M_{A^{\prime}}(G)=\left(L_{A^{\prime}}(G), M\left(G_{k}\right)\right)$. Note that $L M_{A^{\prime}}(G)$ is an object in $S H_{A^{\prime}}^{f}$ and the contravariant functor $L M_{A^{\prime}}: \mathscr{F}_{\mathscr{F}_{A^{\prime}}} \rightarrow S H_{A^{\prime}}^{f}$ is fully faithful and essentially surjective (see [3, Theorem 3.6]). The contravariant functor $L M_{A^{\prime}}$ induces a functor $\widetilde{L M}_{A^{\prime}}$ from $\widetilde{\mathscr{F} \mathscr{F}}_{A^{\prime}}$ to $\widetilde{S H}_{A^{\prime}}^{f}$ which is an anti-equivalence of categories.

\subsection{Finite flat group schemes of order $p$}

We now consider the finite flat group schemes over $A^{\prime}$ of order $p$. Oort and Tate construct certain group schemes over $A^{\prime}$ of order $p$ as follows (see $[8$, Theorem 2]): For any pair $a, b \in A^{\prime}$ with $a \cdot b=p$, define

$$
G_{a, b}=\operatorname{Spec} A^{\prime}[x] /\left(x^{p}-a x\right)
$$

and the comultiplication is given by

$$
\triangle(x)=x \otimes 1+1 \otimes x+\frac{b}{1-p} \sum_{i=1}^{p-1} \frac{x^{i}}{w_{i}} \otimes \frac{x^{p-i}}{w_{p-i}},
$$

in which $w_{1}, \ldots, w_{p-1}$ are certain units of $A^{\prime}$. In particular, $G_{1, p} \simeq \mathbf{Z} / p \mathbf{Z}$ is a constant group scheme and $G_{p, 1} \simeq \mu_{p}$ is a diagonalizable group scheme. Let $a$, $b, c, d$ be elements of $A^{\prime}$ with $a \cdot b=p$ and $c \cdot d=p$. Then $G_{a, b}$ and $G_{c, d}$ are isomorphic to each other if and only if there is a unit $u \in\left(A^{\prime}\right)^{\times}$with

$$
c=u^{p-1} a, \quad d=u^{1-p} b .
$$

According to the classification of finite group schemes of order $p$ due to Oort and Tate, for any group scheme $G$ over $A^{\prime}$ of order $p$, there are $a, b \in A^{\prime}$ with $a \cdot b=p$ such that $G$ is isomorphic to $G_{a, b}$ as group schemes over $A^{\prime}$.

Remark 1.1. For any complete noetherian local ring $R$ with residue characteristic $p>0$, Oort and Tate showed that $(a, b) \mapsto G_{a, b}=$ Spec $R[x] /\left(x^{p}-a x\right)$ gives a bijection between equivalence classes of factorizations $p=a \cdot b$ of $p$ in $R$ and the isomorphism classes of $R$-groups of order $p$.

For $a \in A^{\prime}$, we let $\bar{a}$ denote the residue class in $k$ represented by $a$. According to the Dieudonné theory, finite flat group schemes over $k$ of order $p$ correspond in a one-to-one way to giving a module of length one over the ring $k[F, V]$. For any pair $a, b \in A^{\prime}$ with $a \cdot b=p,\left(G_{a, b}\right)_{k}$ corresponds to the Dieudonné module

$$
k[F, V] / k[F, V] \cdot\left(F-\bar{a}, V-\bar{b}^{1 / p}\right) .
$$

Fix a uniformizer $\pi$ of $A^{\prime}$ and let $v$ be a valuation of $A^{\prime}$ with $v(\pi)=1$. For any pair $a, b \in A^{\prime}$ with $a \cdot b=p$, consider the finite Honda system $L M_{A^{\prime}}\left(G_{a, b}\right)$. Fix $a, b \in A^{\prime}$ with $a \cdot b=p$. For the convention, set $G=G_{a, b}$ and $R=$ $A^{\prime}[x] /\left(x^{p}-a x\right)$. We proceed case by case. 
CASE $v(a)=0$.

The Dieudonné module $M\left(G_{k}\right)$ is isomorphic to $k[F, V]$-module $M=k \mathbf{e}$ with $F \mathbf{e}=\bar{a} \mathbf{e}$ and $V \mathbf{e}=0 . \quad$ In this case, $A^{\prime}$-linear map $\mathscr{F}_{M}: p^{-1} \mathfrak{m} \otimes_{A} M^{(1)} \rightarrow M_{A^{\prime}}$ is an isomorphism. Since $\left(L_{A^{\prime}}(G), M\right)$ consists of a finite Honda system, we see that $A^{\prime}$-submodule $L_{A^{\prime}}(G)$ of $M_{A^{\prime}}$ is trivial.

CASE $v(b)=0$.

The Dieudonné module $M\left(G_{k}\right)$ is isomorphic to $k[F, V]$-module $M=k \mathbf{e}$ with $F \mathbf{e}=0$ and $V \mathbf{e}=\bar{b}^{1 / p} \mathbf{e}$. Since the Cartier dual of $G_{a, b}$ is $G_{b, a}$, we see that $L_{A^{\prime}}(G)=M_{A^{\prime}}$ due to the construction of the dual Honda system (see [3, p. 292293]).

CASE $v(a), v(b)>0$.

Let $v(a)=\ell(1 \leq \ell \leq e-1)$. The Dieudonné module $M\left(G_{k}\right)$ is isomorphic to $k[F, V]$-module $M=k \mathbf{e}$ with $F \mathbf{e}=0$ and $V \mathbf{e}=0$, in which e corresponds to the element $(\ldots, 0,0, x) \in M\left(G_{k}\right)=M\left(R_{k}\right)$. In this case, any $u \in M_{A^{\prime}}$ can be uniquely written in the form

$$
u=\left(1 \otimes \alpha_{0} \mathbf{e}, \frac{\pi}{p} \otimes \alpha_{1} \mathbf{e}+\cdots+\frac{\pi^{p-1}}{p} \otimes \alpha_{e-1} \mathbf{e}\right)
$$

with $\alpha_{0}, \ldots, \alpha_{e-1} \in k$. Easy calculation shows that

$$
w_{R}^{\prime}(u)=\hat{\alpha}_{0} x+\frac{\pi}{p} \hat{\alpha}_{1}^{p} a x+\cdots+\frac{\pi^{e-1}}{p} \hat{\alpha}_{e-1}^{p} a x \quad(\bmod \mathrm{m} R) \in R_{K^{\prime}} / \mathrm{m} R,
$$

with $\hat{\alpha}_{n} \in A^{\prime}$ any lift of $\alpha_{n} \in k$. We can see that $w_{R}^{\prime}(u)=0$ if and only if

$$
\alpha_{1}=\cdots=\alpha_{e-\ell-1}=0 \quad \text { and } \quad \alpha_{0}+\overline{\left(\frac{a \pi^{e-\ell}}{p}\right)} \alpha_{e-\ell}^{p}=0 .
$$

Therefore, by definition, $A^{\prime}$-submodule $L_{A^{\prime}}(G)$ of $M_{A^{\prime}}$ is equal to the set

$$
\left\{\left(1 \otimes \alpha_{0} \mathbf{e}, \frac{\pi^{e-\ell}}{p} \otimes \alpha_{e-\ell} \mathbf{e}+\cdots+\frac{\pi^{p-1}}{p} \otimes \alpha_{e-1} \mathbf{e}\right) \in M_{A^{\prime}} \mid \alpha_{0}+\overline{\left(\frac{a \pi^{e-\ell}}{p}\right)} \alpha_{e-\ell}^{p}=0\right\}
$$

\subsection{Extensions of group schemes of order $p$}

The category $S H_{A^{\prime}}^{f}$ is an abelian category. More precisely, if

$$
\varphi:\left(L_{1}, M_{1}\right) \rightarrow\left(L_{2}, M_{2}\right)
$$

is a morphism in $S H_{A^{\prime}}^{f}$, then $\operatorname{Ker} \varphi=\left(L^{\prime}, M^{\prime}\right)$ and $\operatorname{Coker} \varphi=\left(L^{\prime \prime}, M^{\prime \prime}\right)$ satisfy

$$
M^{\prime}=\operatorname{Ker}\left[M_{1} \rightarrow M_{2}\right], \quad M^{\prime \prime}=\operatorname{Coker}\left[M_{1} \rightarrow M_{2}\right]
$$


and

$$
L^{\prime}=\left(M^{\prime}\right)_{A^{\prime}} \cap L_{1}, \quad L^{\prime \prime}=\operatorname{image}\left[L_{2} \hookrightarrow\left(M_{2}\right)_{A^{\prime}} \rightarrow\left(M^{\prime \prime}\right)_{A^{\prime}}\right],
$$

and the natural map Coker $\left[L_{1} \rightarrow L_{2}\right] \rightarrow L^{\prime \prime}$ is an isomorphism (see [3, Theorem 4.3]). Let $\mathfrak{M}_{1}, \mathfrak{M}_{2} \in \widetilde{S H}_{A^{\prime}}^{f}$. Consider the group $\operatorname{Ext}_{\widetilde{S H}_{A^{\prime}}^{f}}^{1}\left(\mathfrak{M}_{2}, \mathfrak{M}_{1}\right)$ of equivalence classes of exact sequences $0 \rightarrow \mathfrak{M}_{1} \rightarrow \mathfrak{M} \rightarrow \mathfrak{M}_{2} \rightarrow 0$ in the category $\widetilde{S H}_{A^{\prime}}^{f}$. Put $\mathfrak{M}_{1}=\left(L_{1}, M_{1}\right), \mathfrak{M}=(L, M)$ and $\mathfrak{M}_{2}=\left(L_{2}, M_{2}\right)$. Then the above sequence is exact if and only if the induced sequences of $D_{k}$-modules $0 \rightarrow M_{1} \rightarrow M \rightarrow$ $M_{2} \rightarrow 0$ and of $A^{\prime}$-modules $0 \rightarrow L_{1} \rightarrow L \rightarrow L_{2} \rightarrow 0$ have this property.

Let $a, b, c, d$ be elements of $A^{\prime}$ with $a \cdot b=p$ and $c \cdot d=p$. Using the antiequivalence $\widetilde{L M}_{A^{\prime}}: \widetilde{\mathscr{F}}_{A^{\prime}} \rightarrow \widetilde{S H}_{A^{\prime}}^{f}$, we obtain that

$$
\operatorname{Ext}_{\widetilde{F}_{\mathscr{F}_{A^{\prime}}}}^{1}\left(G_{a, b}, G_{c, d}\right) \simeq \operatorname{Ext}_{\widetilde{S H}_{A^{\prime}}^{f}}^{1}\left(L M_{A^{\prime}}\left(G_{c, d}\right), L M_{A^{\prime}}\left(G_{a, b}\right)\right)
$$

We now consider the group $\operatorname{Ext}_{\widetilde{S H}_{A^{\prime}}^{f}}^{1}\left(L M_{A^{\prime}}\left(G_{c, d}\right), L M_{A^{\prime}}\left(G_{a, b}\right)\right)$. Set $L M_{A^{\prime}}\left(G_{a, b}\right)$ $=\left(L_{1}, M_{1}\right)$ and $L M_{A^{\prime}}\left(G_{c, d}\right)=\left(L_{2}, M_{2}\right)$. Fix

$$
(L, M) \in \operatorname{Ext}_{\widetilde{S H}_{A^{\prime}}^{f}}^{1}\left(\left(L_{2}, M_{2}\right),\left(L_{1}, M_{1}\right)\right) .
$$

Since $M_{1}$ and $M_{2}$ are $k[F, V]$-modules of length one, we write $M_{1}=k \mathbf{e}_{1}$ and $M_{2}=k \mathbf{e}_{2}$ as before. Then we can choose a basis $\left\{\mathbf{e}, \mathbf{e}^{\prime}\right\}$ for $M$ as a $k$-vector space as follows:

$$
0 \rightarrow M_{1} \stackrel{f}{\rightarrow} M \stackrel{g}{\rightarrow} M_{2} \rightarrow 0,
$$

where $f\left(\mathbf{e}_{1}\right)=\mathbf{e}, g(\mathbf{e})=0$ and $g\left(\mathbf{e}^{\prime}\right)=\mathbf{e}_{2}$. Since the exact sequence

$$
0 \rightarrow\left(M_{1}\right)_{A^{\prime}} \rightarrow M_{A^{\prime}} \rightarrow\left(M_{2}\right)_{A^{\prime}} \rightarrow 0
$$

is split as $A^{\prime}$-modules, the $A^{\prime}$-submodule $L$ of $M_{A^{\prime}}$ is uniquely determined by $L_{1}$ and $L_{2}$. Therefore it suffices to consider the structure of $k[F, V]$-module on $M$. If the actions $F$ and $V$ on $M$ are given by

$$
\begin{aligned}
& F \mathbf{e}=\alpha \mathbf{e}+\beta \mathbf{e}^{\prime}, \quad V \mathbf{e}=\alpha^{\prime} \mathbf{e}+\beta^{\prime} \mathbf{e}^{\prime}, \\
& F \mathbf{e}^{\prime}=\gamma \mathbf{e}+\delta \mathbf{e}^{\prime}, \quad V \mathbf{e}^{\prime}=\gamma^{\prime} \mathbf{e}+\delta^{\prime} \mathbf{e}^{\prime},
\end{aligned}
$$

with $\alpha, \beta, \gamma, \delta, \alpha^{\prime}, \beta^{\prime}, \gamma^{\prime}, \delta^{\prime} \in k$, we simply write

$$
F=\left(\begin{array}{ll}
\alpha & \beta \\
\gamma & \delta
\end{array}\right), \quad V=\left(\begin{array}{ll}
\alpha^{\prime} & \beta^{\prime} \\
\gamma^{\prime} & \delta^{\prime}
\end{array}\right) .
$$

We proceed case by case.

CASE $v(a)=v(c)=0$.

Since the sequence (1) is exact as $k[F, V]$-modules, we obtain that the actions of $F$ and $V$ on $M$ are given by

$$
F=\left(\begin{array}{cc}
\bar{a} & \alpha \\
0 & \bar{c}
\end{array}\right), \quad V=\left(\begin{array}{cc}
0 & \beta \\
0 & 0
\end{array}\right)
$$


with $\alpha, \beta \in k$. Since $F V=V F=0$ on $M$, we get $\beta=0$. Therefore the actions of $F$ and $V$ on $M$ are given by

$$
F=\left(\begin{array}{cc}
\bar{a} & \alpha \\
0 & \bar{c}
\end{array}\right), \quad V=\left(\begin{array}{ll}
0 & 0 \\
0 & 0
\end{array}\right),
$$

with $\alpha \in k$. Note that by these actions on $M,(L, M)$ becomes a finite Honda system.

CASE $v(a)=v(d)=0$.

A similar calculation shows that the actions of $F$ and $V$ on $M$ are given by

$$
F=\left(\begin{array}{cc}
\bar{a} & \alpha \\
0 & 0
\end{array}\right), \quad V=\left(\begin{array}{cc}
0 & 0 \\
0 & \bar{d}^{1 / p}
\end{array}\right),
$$

with $\alpha \in k$.

CASE $v(a)=0,1 \leq v(c) \leq e-1$.

A similar calculation shows that the actions of $F$ and $V$ on $M$ are given by

$$
F=\left(\begin{array}{cc}
\bar{a} & \alpha \\
0 & 0
\end{array}\right), \quad V=\left(\begin{array}{ll}
0 & 0 \\
0 & 0
\end{array}\right),
$$

with $\alpha \in k$.

CASE $1 \leq v(a), v(c) \leq e-1$.

Since the sequence (1) is exact as $k[F, V]$-modules, we obtain that the actions of $F$ and $V$ on $M$ are given by

$$
F=\left(\begin{array}{ll}
0 & \alpha \\
0 & 0
\end{array}\right), \quad V=\left(\begin{array}{ll}
0 & \beta \\
0 & 0
\end{array}\right),
$$

with $\alpha, \beta \in k$. Since the canonical $k$-linear map $L / m L \rightarrow \operatorname{Coker} \mathscr{F}_{M}$ is an isomorphism, we get $\alpha=0$. Therefore the actions of $F$ and $V$ on $M$ are given by

$$
F=\left(\begin{array}{ll}
0 & 0 \\
0 & 0
\end{array}\right), \quad V=\left(\begin{array}{ll}
0 & \beta \\
0 & 0
\end{array}\right),
$$

with $\beta \in k$. Note that by these actions on $M,(L, M)$ becomes a finite Honda system.

Considering the Cartier dual, we get the following results:

THEOREM 1.2. Let $q=p^{f}$ and assume $k=\mathbf{F}_{q}$. Let $a, b, c, d$ be elements of $A^{\prime}$ with $a \cdot b=p$ and $c \cdot d=p$. 
(1) If $v(a) \neq 0$ and $v(c)=0$, we have $\operatorname{Ext}_{\mathscr{F}_{\mathscr{F}^{\prime}}}^{1}\left(G_{a, b}, G_{c, d}\right)=0$.

(2) If $v(a)=0$ or $v(c) \neq 0$, we have

$$
\operatorname{dim}_{\mathbf{F}_{p}} \operatorname{Ext}_{\widetilde{F}_{\mathscr{F}_{A}}^{\prime}}^{1}\left(G_{a, b}, G_{c, d}\right)=f .
$$

Proof. (1) In this case, we see that $G_{a, b}$ is connected while $G_{c, d}$ is étale. This implies that $\operatorname{Ext}_{\mathscr{F}_{\mathscr{F}} \mathscr{F}^{\prime}}^{1}\left(G_{a, b}, G_{c, d}\right)=0$.

(2) This follows from calculations above.

\section{Extensions of $\mu_{p}$ by $\mathbf{Z} / p \mathbf{Z}$ and of $\mathbf{Z} / p \mathbf{Z}$ by $\mu_{p}$}

Let $K$ be a number field and $p$ be a prime number. In this section, we consider the groups of extensions of a diagonalizable group scheme $\mu_{p}$ by a constant group scheme $\mathbf{Z} / p \mathbf{Z}$ and of extensions of $\mathbf{Z} / p \mathbf{Z}$ by $\mu_{p}$ over the ring of integers $\mathcal{O}_{K}$ of $K$.

\subsection{An equivalence of categories}

Let $R$ be a Noetherian ring, let $p \in R$ and let $\underline{G r}_{R}$ denote the category of finite flat $R$-group schemes. Let

$$
\hat{R}=\lim _{\longleftarrow} R / p^{n} R
$$

and let $\underline{C}$ be the category of triples $\left(G_{1}, G_{2}, \theta\right)$ where $G_{1}$ is a finite flat $\hat{R}$-group scheme, $G_{2}$ is a finite flat $R[1 / p]$-group scheme and

$$
\theta: G_{1} \otimes_{\hat{R}} \hat{R}[1 / p] \rightarrow G_{2} \otimes_{R[1 / p]} \hat{R}[1 / p]
$$

is an isomorphism of $\hat{R}[1 / p]$-group schemes. Morphisms in $\underline{C}$ are pairs of morphisms of group schemes that are compatible with the morphisms $\theta$. The functor $\underline{G r}_{R} \rightarrow \underline{C}$ that sends an $R$-group scheme $G$ to the triple

$$
\left(G \otimes_{R} \hat{R}, G \otimes_{R} R[1 / p], \mathrm{id} \otimes_{R} \hat{R}[1 / p]\right)
$$

is an equivalence of categories (see [1, Theorem 2.6]). The equivalence of categories above gives the following result (see [9, Corollary 2.4]):

Theorem 2.1. Let $G$ and $H$ be two finite flat group schemes over $R$. There is a natural exact "Mayer-Vietoris" sequence

$$
\begin{aligned}
0 & \rightarrow \operatorname{Hom}_{R}(G, H) \rightarrow \operatorname{Hom}_{\hat{R}}(G, H) \times \operatorname{Hom}_{R[1 / p]}(G, H) \rightarrow \operatorname{Hom}_{\hat{R}[1 / p]}(G, H) \\
& \stackrel{\delta}{\rightarrow} \operatorname{Ext}_{R}^{1}(G, H) \rightarrow \operatorname{Ext}_{\hat{R}}^{1}(G, H) \times \operatorname{Ext}_{R[1 / p]}^{1}(G, H) \rightarrow \operatorname{Ext}_{\hat{R}[1 / p]}^{1}(G, H),
\end{aligned}
$$

where $\delta$ maps an $\hat{R}[1 / p]$-morphism $\varphi: G \rightarrow H$ to the extension of $G$ by $H$ that corresponds to the triple

$$
\left((H \times G)_{\hat{R}},(H \times G)_{R[1 / p]}, \theta\right),
$$

where $\theta(h, g)=(h+\varphi(g), g)$. 
In the applications, $R$ is the ring of integers of a number field $K$, the element $p$ is a prime number, and $G$ and $H$ are $p$-group schemes. Then $G$ and $H$ are étale over $R[1 / p]$ and we can identify them with their Galois modules. The Galois action is unramified outside $p$. The ring $\hat{R}$ is a finite product of finite extensions of $\mathbf{Z}_{p}$. Finally, the $\operatorname{ring} \hat{R}[1 / p] \cong K \otimes \mathbf{Q}_{p}$ is a product of $p$-adic fields. Over each of these fields the group schemes can be identified with their local Galois modules.

2.2. Extensions of $\mu_{p}$ by $\mathbf{Z} / p \mathbf{Z}$ and of $\mathbf{Z} / p \mathbf{Z}$ by $\mu_{p}$

Let $p$ be a prime number and let $\zeta_{p}$ denote a primitive $p$-th root of unity. Let $K$ be a number field and let $\mathcal{O}_{K}$ and $\mathcal{O}_{K}^{\times}$denote its ring of integers and its group of units. For each prime $\mathfrak{p}$ of $K$ over $p$, let $e_{\mathfrak{p}}$ and $f_{\mathfrak{p}}$ denote the ramification index and the residue degree of $\mathfrak{p}$ in the extension $K / \mathbf{Q}$, respectively.

THEOREM 2.2. Let $K$ be a number field and let $p$ be a prime number. Suppose that $p$ does not divide the class number of $K\left(\zeta_{p}\right)$ and the ramification index $e_{\mathfrak{p}}$ satisfies $e_{\mathfrak{p}}<p-1$ for all primes $\mathfrak{p}$ of $K$ over $p$. Then we have

(1) $\operatorname{Ext}_{\mathfrak{O}_{K}}^{1}\left(\mu_{p}, \mathbf{Z} / p \mathbf{Z}\right)=0$.

(2) $\operatorname{dim}_{\mathbf{F}_{p}} \operatorname{Ext}_{\mathscr{O}_{K}, p}^{1}\left(\mathbf{Z} / p \mathbf{Z}, \mu_{p}\right) \leq \sum_{\mathfrak{p} \mid p} f_{\mathfrak{p}}$.

Here the index ' $p$ ' means 'the p-torsion part'.

Proof. (1) This is proved by Schoof (see [9, Theorem 2.6]).

(2) Since $e_{\mathfrak{p}}<p-1$ for all primes $\mathfrak{p}$ over $p$, the $p$-th roots of unity are not contained in any of the completions at $\mathfrak{p}$. This implies that $\operatorname{Hom}_{\hat{\mathscr{O}}_{K}[1 / p]}\left(\mathbf{Z} / p \mathbf{Z}, \mu_{p}\right)=0$. Therefore, by Theorem 2.1, there is an exact sequence

$$
\begin{aligned}
0 & \rightarrow \operatorname{Ext}_{\hat{O}_{K}}^{1}\left(\mathbf{Z} / p \mathbf{Z}, \mu_{p}\right) \rightarrow \operatorname{Ext}_{\hat{\mathscr{O}}_{K}}^{1}\left(\mathbf{Z} / p \mathbf{Z}, \mu_{p}\right) \times \operatorname{Ext}_{\mathscr{O}_{K}[1 / p]}^{1}\left(\mathbf{Z} / p \mathbf{Z}, \mu_{p}\right) \\
& \rightarrow \operatorname{Ext}_{\hat{\mathscr{O}}_{K}[1 / p]}^{1}\left(\mathbf{Z} / p \mathbf{Z}, \mu_{p}\right) .
\end{aligned}
$$

Fix $G \in \operatorname{Ext}_{\mathcal{O}_{K}, p}^{1}\left(\mathbf{Z} / p \mathbf{Z}, \mu_{p}\right)$, which is split over $\hat{\mathcal{O}}_{K}$. Since $G$ is killed by $p$ and split over $\hat{\mathcal{O}}_{K}$, the extension $L$ obtained by adjoining the points of $G$ to $K\left(\zeta_{p}\right)$ has degree dividing $p$ and is unramified at all primes. Since $p$ does not divide the class number of $K\left(\zeta_{p}\right)$, it follows that $L=K\left(\zeta_{p}\right)$. Therefore $G$ is split over $\mathcal{O}_{K}[1 / p]$ and hence $G$ is split over $\mathcal{O}_{K}$. Therefore we have

$$
\operatorname{dim}_{\mathbf{F}_{p}} \operatorname{Ext}_{\hat{O}_{K}, p}^{1}\left(\mathbf{Z} / p \mathbf{Z}, \mu_{p}\right) \leq \operatorname{dim}_{\mathbf{F}_{p}} \operatorname{Ext}_{\hat{\mathfrak{O}}_{K}, p}^{1}\left(\mathbf{Z} / p \mathbf{Z}, \mu_{p}\right) .
$$

This completes the proof by Theorem 1.2.

The group $\operatorname{Ext}_{\mathcal{O}_{K}, p}^{1}\left(\mathbf{Z} / p \mathbf{Z}, \mu_{p}\right)$ may be non-trivial when the ring $\mathcal{O}_{K}$ contains certain units. The group schemes constructed by Katz and Mazur provide examples of such non-trivial extensions (see [6, Interlude (8.7)]). Let $R$ be a ring and let $\varepsilon \in R^{\times}$. Consider the $R$-algebra

$$
A=\bigoplus_{i=0}^{p-1} R\left[X_{i}\right] /\left(X_{i}^{p}-\varepsilon^{i}\right) .
$$


For any $R$-algebra $S$ with connected spectrum, the $S$-points of $T_{\varepsilon}=\operatorname{Spec} A$ are pairs $(s, i)$ with $0 \leq i \leq p-1$ and $s \in S$ satisfying $s^{p}=\varepsilon^{i}$. The scheme $T_{\varepsilon}$ is a finite flat $R$-algebra scheme with multiplication of two pairs $(s, i)$ and $(t, j)$ given by

$$
(s, i) \cdot(t, j)= \begin{cases}(s t, i+j) & \text { if } i+j<p, \\ (s t / \varepsilon, i+j-p) & \text { if } i+j \geq p .\end{cases}
$$

The group scheme $T_{\varepsilon}$ is killed by $p$. The projection $A \rightarrow R\left[X_{0}\right] /\left(X_{0}^{p}-1\right)$ induces a closed flat immersion of $\mu_{p}$ in $T_{\varepsilon}$. There is an exact sequence

$$
0 \rightarrow \mu_{p} \rightarrow T_{\varepsilon} \rightarrow \mathbf{Z} / p \mathbf{Z} \rightarrow 0 .
$$

Two extensions $T_{\varepsilon}$ and $T_{\varepsilon^{\prime}}$ are isomorphic whenever $\varepsilon / \varepsilon^{\prime}$ is a $p$-th power. If $R$ is a field, the points of $T_{\varepsilon}$ generate the field extension $R\left(\zeta_{p}, \sqrt[p]{\varepsilon}\right)$.

\section{Finite flat group schemes of prime order over certain number fields}

Let $K$ be a number field. Let $\mathcal{O}_{K}$ and $\mathcal{O}_{K}^{\times}$denote its ring of integers and its group of units. We shall review here the classification of group schemes of prime order over $\mathcal{O}_{K}$ due to Oort and Tate (see [8]). Fix a prime number $p$. Let $M$ be the set of non-generic points of $\operatorname{Spec}\left(\mathcal{O}_{K}\right)$ and let $M_{p}$ denote the set of $\mathfrak{p} \in M$ such that $\mathfrak{p}$ divides $p$. For each $\mathfrak{p} \in M$, let $\mathcal{O}_{K, \mathfrak{p}}$ denote the completion of $\mathcal{O}_{K}$ at $\mathfrak{p}$, let $K_{\mathfrak{p}}$ denote the field of fractions of $\mathcal{O}_{K, \mathfrak{p}}$, and let $U_{\mathfrak{p}}$ denote the group of units in $\mathcal{O}_{K, \mathfrak{p}}$. For each $\mathfrak{p} \in M_{p}$, we let $v_{\mathfrak{p}}$ denote the corresponding normalized discrete valuation of $K$, let $k_{\mathfrak{p}}$ denote the residue field of $\mathcal{O}_{K, \mathfrak{p}}$ and let $u \mapsto \bar{u}$ denote the residue class $\operatorname{map} \mathcal{O}_{K, \mathfrak{p}} \rightarrow k_{\mathfrak{p}}$. Let $C_{K}$ denote the idèle class group of $K$. Let $E$ denote the functor which associates with commutative ring $R$ with unity the set $E(R)$ of isomorphism classes of $R$-groups of order $p$. Then they showed that the square

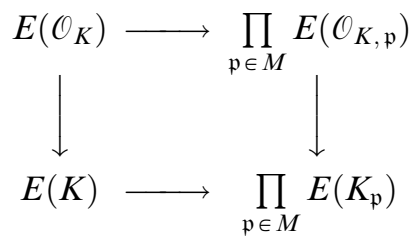

is cartesian (see [8, Lemma 4]). Using class field theory, there are canonical bijections

$$
\begin{aligned}
E(K) & \simeq \operatorname{Hom}_{\text {cont }}\left(C_{K}, \mathbf{F}_{p}^{\times}\right), \\
E\left(K_{\mathfrak{p}}\right) & \simeq \operatorname{Hom}_{\text {cont }}\left(K_{\mathfrak{p}}^{\times}, \mathbf{F}_{p}^{\times}\right) \quad(\mathfrak{p} \in M) \quad \text { and } \\
E\left(\mathcal{O}_{K, \mathfrak{p}}\right) & \simeq \operatorname{Hom}_{\text {cont }}\left(K_{\mathfrak{p}}^{\times} / U_{\mathfrak{p}}, \mathbf{F}_{p}^{\times}\right) \quad\left(\mathfrak{p} \in M \backslash M_{p}\right),
\end{aligned}
$$

where Hom $_{\text {cont }}$ denotes the continuous homomorphisms (see [8, Lemma 6]). Via these bijections the arrows in the diagram (2) are induced by the canonical 
homomorphisms $K_{\mathfrak{p}}^{\times} \rightarrow C_{K}$ and $K_{\mathfrak{p}}^{\times} \rightarrow K_{\mathfrak{p}}^{\times} / U_{\mathfrak{p}}$. If $G$ is an $\mathcal{O}_{K}$-group scheme of order $p$, we shall denote by $\psi^{G} \in \operatorname{Hom}_{\text {cont }}\left(C_{K}, \mathbf{F}_{p}^{\times}\right)$the idéle class character determined by $G \otimes_{\mathcal{O}_{K}} K$, and by $\psi_{\mathrm{p}}^{G}$ the corresponding character of $K_{\mathfrak{p}}^{\times}$, for each $\mathfrak{p} \in M$. For each $\mathfrak{p} \in M_{p}$, we let $n_{\mathfrak{p}}^{G}=v(a)$, where $a \in \mathcal{O}_{K, \mathfrak{p}}$ is such that $G \otimes_{\mathcal{O}_{K}} \mathcal{O}_{K, \mathfrak{p}} \simeq\left(G_{a, b}\right)_{\mathcal{O}_{K, \mathfrak{p}}}$ in the notation of remark 1.1. Note that $a$ is determined up to $U_{\mathfrak{p}}^{p-1}$ by $G \otimes_{\mathcal{O}_{K}} \mathcal{O}_{K, \mathfrak{p}}$, hence $n_{\mathfrak{p}}^{G}$ is uniquely determined by $G$. They showed the following theorem (see [8, Theorem 3]):

THEOREM 3.1. The map $G \mapsto\left(\psi^{G},\left(n_{\mathfrak{p}}^{G}\right)_{\mathfrak{p} \in M_{p}}\right)$ gives a bijection between the isomorphism classes of $\mathcal{O}_{K}$-groups of order $p$ and the systems $\left(\psi,\left(n_{\mathfrak{p}}\right)_{\mathfrak{p} \in M_{p}}\right)$ consisting of a continuous homomorphism $\psi: C_{K} \rightarrow \mathbf{F}_{p}^{\times}$and for each $\mathfrak{p} \in M_{p}$ an integer $n_{\mathfrak{p}}$ such that $0 \leq n_{\mathfrak{p}} \leq v_{\mathfrak{p}}(p)$, which satisfy the following conditions:

(1) For $\mathfrak{p} \in M \backslash M_{p}, \psi$ is unramified at $\mathfrak{p}$, i.e. $\psi_{\mathfrak{p}}\left(U_{\mathfrak{p}}\right)=1$,

(2) For $\mathfrak{p} \in M_{p}, \psi_{\mathfrak{p}}(u)=\left(\mathrm{Nm}_{k_{\mathfrak{p}} / \mathbf{F}_{p}}(\bar{u})\right)^{-n_{\mathfrak{p}}}$.

Here $\psi_{\mathfrak{p}}: K_{\mathfrak{p}}^{\times} \rightarrow \mathbf{F}_{p}^{\times}$denotes the local character induced by $\psi$ via the canonical map $K_{\mathfrak{p}}^{\times} \rightarrow C_{K}$ and $\mathrm{Nm}_{k_{\mathrm{p}} / \mathbf{F}_{p}}$ denotes the norm map.

For a given family of integers $\left(n_{\mathfrak{p}}\right)_{\mathfrak{p} \in M_{p}}$, there is either no idèle class character $\psi$ satisfying (1) and (2) of Theorem 3.1, or the set of all idèle characters is a principal homogeneous space under the group of homomorphisms of the ideal class group $\mathrm{Cl}(K)$ of $K$ into $\mathbf{F}_{p}^{\times}$. Therefore, if the class number of $K$ is prime to $(p-1)$, there is at most one $\psi$ for each family $\left(n_{\mathfrak{p}}\right)_{\mathfrak{p} \in M_{p}}$.

\subsection{Imaginary quadratic fields of class number one}

Let $K=\mathbf{Q}(\sqrt{m})$ be a quadratic field, where $m$ is a square-free integer. Let $\zeta_{n}$ denote a primitive $n$-th root of unity. Set

$$
N= \begin{cases}|m| & \text { if } m \equiv 1(\bmod 4), \\ 4|m| & \text { if } m \equiv 2,3(\bmod 4) .\end{cases}
$$

We have $K \subset \mathbf{Q}\left(\zeta_{N}\right)$. For an odd prime $p$ and integer $a$ not divisible by $p$, we let $(a / p)$ denote the quadratic residue symbol. We give here a lemma which we use later.

Lemma 3.2. Let $p$ be an odd prime number. Let $n$ denote the degree of the extension $\mathbf{Q}\left(\zeta_{p \cdot N}\right) / K\left(\zeta_{p}\right)$. Suppose $p$ divides neither $n$ nor the class number of the cyclotomic field $\mathbf{Q}\left(\zeta_{p \cdot N}\right)$. Then the class number of the field $K\left(\zeta_{p}\right)$ is not divisible by $p$.

Proof. If the class number of the field $K\left(\zeta_{p}\right)$ is divisible by $p$, then there exists an abelian extension $H / K\left(\zeta_{p}\right)$ which is unramified everywhere of $p$-power degree. Since $p$ is prime to $n$, the abelian extension $H \cdot \mathbf{Q}\left(\zeta_{p \cdot N}\right) / \mathbf{Q}\left(\zeta_{p \cdot N}\right)$ is unramified everywhere of $p$-power degree. By assumption, this is a contadiction. 
Assume that $K$ is an imaginary quadratic field of class number one. As is well known, there are nine imaginary quadratic fields of class number one. These fields are

$$
\begin{gathered}
\mathbf{Q}(\sqrt{-1}), \mathbf{Q}(\sqrt{-2}), \mathbf{Q}(\sqrt{-3}), \mathbf{Q}(\sqrt{-7}), \mathbf{Q}(\sqrt{-11}) \\
\mathbf{Q}(\sqrt{-19}), \mathbf{Q}(\sqrt{-43}), \mathbf{Q}(\sqrt{-67}), \mathbf{Q}(\sqrt{-163}) .
\end{gathered}
$$

We consider the finite flat group schemes over $\mathcal{O}_{K}$ of prime order.

Proposition 3.3. Let $p$ be an odd prime number such that $p$ does not ramify in $K$. Then the only group schemes of order $p$ over $\mathcal{O}_{K}$ are $\mu_{p}$ and $\mathbf{Z} / p \mathbf{Z}$.

Proof. In the case that $(m / p)=-1$, this is proved by Oort and Tate (see $[8$, Corollary of Theorem 3]). In the case that $(m / p)=1$, there are two primes $\mathfrak{p}, \overline{\mathfrak{p}}$ in $K$ over $p$. We introduce the usual notation. For any integral ideal $\mathfrak{a}$ of $K$, we let $U(\mathfrak{a})$ be the subgroup of the idéle group $\mathbf{A}_{K}^{\times}$of $K$ defined by

$$
U(\mathfrak{a})=\left\{s \in \mathbf{A}_{K}^{\times} \mid s_{\mathfrak{p}} \in U_{\mathfrak{p}} \text { and } s_{\mathfrak{p}} \equiv 1\left(\bmod \mathfrak{a} \mathcal{O}_{K, \mathfrak{p}}\right) \text { for all primes } \mathfrak{p}\right\} \text {. }
$$

Let $\bar{U}(\mathfrak{a})$ be the image of $U(\mathfrak{a})$ of the canonical map $\mathbf{A}_{K}^{\times} \rightarrow C_{K}$ and set

$$
\mathrm{Cl}(K, \mathfrak{a})=C_{K} / \bar{U}(\mathfrak{a}) .
$$

Since the field $K$ has no real places, there is an exact sequence

$$
1 \rightarrow\left(\mathcal{O}_{K} / \mathfrak{a}\right)^{\times} / \operatorname{img} \mathcal{O}_{K}^{\times} \rightarrow \mathrm{Cl}(K, \mathfrak{a}) \rightarrow \mathrm{Cl}(K) \rightarrow 0,
$$

where $\operatorname{img} \mathcal{O}_{K}^{\times}$denotes the image of $\mathcal{O}_{K}^{\times}$of the natural map $\mathcal{O}_{K}^{\times} \rightarrow\left(\mathcal{O}_{K} / \mathfrak{a}\right)^{\times}$.

For the family $\left(n_{\mathfrak{p}}, n_{\mathfrak{p}}\right)=(1,0)$, we assume that there is a continuous homomorphism $\psi: C_{K} \rightarrow \mathbf{F}_{p}^{\times}$satisfying the conditions (1) and (2) of Theorem 3.1. Then we have a surjective homomorphism $\bar{\psi}: \mathrm{Cl}(K, \mathfrak{p}) \rightarrow \mathbf{F}_{p}^{\times}$induced by $\psi$. Since the class number of $K$ is equal to 1 , we have an isomorphism

$$
\left(\mathcal{O}_{K} / \mathfrak{p}\right)^{\times} / \operatorname{img} \mathcal{O}_{K}^{\times} \simeq \mathrm{Cl}(K, \mathfrak{p}) .
$$

Since $\pm 1 \in \mathcal{O}_{K}^{\times}$, this is a contradiction. In a similar way, there is no idèle class character $\psi$ satisfying the conditions (1) and (2) of Theorem 3.1 for the family $\left(n_{\mathfrak{p}}, n_{\overline{\mathfrak{p}}}\right)=(0,1)$. Therefore the $\mathcal{O}_{K}$-group schemes of order $p$ are $\mu_{p}$ and $\mathbf{Z} / p \mathbf{Z}$.

An abelian variety over a number field $k$ is said to have good reduction if it has good reduction at every finite place of the ring of integers of $k$. We now consider an abelian variety $A$ over $K$ with good reduction. Recently, Schoof proved that for every conductor $f \in\{1,3,4,5,7,8,9,12\}$ there do not exist nonzero abelian varieties over $\mathbf{Q}\left(\zeta_{f}\right)$ with good reduction (see [9, Theorem 1.1]). Assuming the Generalized Riemann Hypothesis (GRH), he proved the same results when $f=11$ and 15 (see [9, Theorem 1.1]). Therefore there do not exist non-zero abelian varieties over $K=\mathbf{Q}(\sqrt{m})$ with good reduction everywhere for $m \in\{-1,-2,-3,-7,-11\}$ under the GRH. 
Let $K=\mathbf{Q}(\sqrt{m})$ be an imaginary quadratic field with class number one. Let $p$ be an odd prime number such that $p$ does not ramify in $K$. We now consider an abelian variety $A$ over $K$ with bad reduction only at the primes of $K$ over $p$. Let $\mathscr{A}$ be the Néron model of $A$ over $\mathcal{O}_{K}$. Since $A$ has bad reduction only at the primes of $K$ over $p$, note that $\mathscr{A}\left[p^{n}\right]$ is a finite flat group scheme over $\mathcal{O}_{K}[1 / p]$. Let $\mathfrak{p}$ be a prime of $K$ over $p$. Note that any finite flat group scheme over $K_{\mathfrak{p}}$ of $p$-power order admits a prolongation over $\mathcal{O}_{K, \mathfrak{p}}$ (see [5, Théorème 3.3.3]). Therefore there is a finite flat group scheme $G$ over $\mathcal{O}_{K}$ such that $G$ is isomorphic to $\mathscr{A}\left[p^{n}\right]$ over $\mathcal{O}_{K}[1 / p]$, using the equivalence of categories between the category $\underline{G r}_{\mathcal{O}_{K}}$ of $\mathcal{O}_{K}$-group schemes and the category $\underline{C}$ of triples $\left(G_{1}, G_{2}, \theta\right)$ where $G_{1}$ is a finite flat $\hat{\mathcal{O}}_{K}$-group scheme, $G_{2}$ is a finite flat $\mathcal{O}_{K}[1 / p]$-group scheme and $\theta: G_{1} \otimes \hat{\mathcal{O}}_{K}[1 / p] \rightarrow G_{2} \otimes \hat{\mathcal{O}}_{K}[1 / p]$ is an isomorphism of $\hat{\mathcal{O}}_{K}[1 / p]$-group schemes. For the convention, we simply write $\mathscr{A}\left[p^{n}\right]$ for $G$.

LemMa 3.4. Let $p$ be an odd prime number such that $p$ does not ramify in $K$ and $(m / p)=1$. Assume $A$ has complex multiplication over $K$. Then the finite flat group scheme $\mathscr{A}\left[p^{n}\right]$ admits a filtration

$$
0=G_{s} \subset G_{s-1} \subset \cdots \subset G_{1} \subset G_{0}=\mathscr{A}\left[p^{n}\right]
$$

by closed flat subgroup schemes such that successive subquotients $G_{i} / G_{i+1}$ have order $p$.

Proof. Let $G$ be a simple subgroup scheme of $\mathscr{A}\left[p^{n}\right]$. Set $L=K(G(\bar{K}))$ and let $S_{p}$ be the $p$-Sylow subgroup of $\operatorname{Gal}(L / K)$. Since $A$ has complex multiplication over $K$, it follows that the $\operatorname{group} \operatorname{Gal}\left(K\left(A\left[p^{n}\right]\right) / K\right)$ is abelian (see [10, Corollar 2 of Theorem 5]).

Therefore the group $\operatorname{Gal}(L / K)$ is abelian and hence the $S_{p}$-fixed points $G(\bar{K})^{S_{p}}$ is a $\operatorname{Gal}(L / K)$-submodule of $G(\bar{K})$. Since

$$
\# G(\bar{K}) \equiv \# G(\bar{K})^{S_{p}} \quad(\bmod p),
$$

we see that $G(\bar{K})^{S_{p}}$ is non-trivial. Since $G$ is simple, it follows that $G(\bar{K})=$ $G(\bar{K})^{S_{p}}$. Let $L^{\prime}$ be the fixed field of $S_{p}$. Then $G(\bar{K})$ is a $\operatorname{Gal}\left(L^{\prime} / K\right)$-module. By assumption, there are two primes $\mathfrak{p}, \bar{p}$ of $K$ over $p$. If $v$ is a nonarchimedean place, set $U_{v}^{(n)}=\left\{x \in U_{v} \mid v(x-1) \geq n\right\}$. Let $\mathscr{N}$ be the norm subgroup of $\mathbf{A}_{K}^{\times}$defined by

$$
\mathscr{N}=\left(U_{\mathfrak{p}}^{(1)} \times U_{\overline{\mathfrak{p}}}^{(1)} \times \prod_{v \neq \mathfrak{p}, \overline{\mathfrak{p}}} U_{v}\right) \cdot \mathbf{K}^{\times},
$$

where $U_{v}=\mathbf{C}^{\times}$for the archimedean places $v$ and $\mathbf{K}^{\times}$is the image of $K^{\times}$on the diagonal. By class field theory, there is a surjection $\mathbf{A}_{K}^{\times} / \mathscr{N} \rightarrow \operatorname{Gal}\left(L^{\prime} / K\right)$. Let $V_{K}$ be the image of the global units of $K$ in

$$
\Gamma=U_{\mathfrak{p}} / U_{\mathfrak{p}}^{(1)} \times U_{\overline{\mathfrak{p}}} / U_{\overline{\mathfrak{p}}}^{(1)} .
$$


Then we have the exact sequence

$$
0 \rightarrow \Gamma / V_{K} \rightarrow \mathbf{A}_{K}^{\times} / \mathscr{N} \rightarrow \mathbf{A}_{K}^{\times} /\left(\left(\prod_{v} U_{v}\right) \cdot \mathbf{K}^{\times}\right) \rightarrow 0 .
$$

Here, the last quotient is isomorphic to the ideal class group of $K$, which is trivial. Therefore the group $\operatorname{Gal}\left(L^{\prime} / K\right)$ has exponent dividing $p-1$. The $\mathbf{F}_{p}\left[\operatorname{Gal}\left(L^{\prime} / K\right)\right]$-module $G(\bar{K})$ is therefore a product of 1-dimensional eigenspaces. Since $G$ is simple, there is only one such eigenspaces and $G$ has order $p$. In a similar way, the finite flat group scheme $\mathscr{A}\left[p^{n}\right]$ admits a filtration

$$
0=G_{s} \subset G_{s-1} \subset \cdots \subset G_{1} \subset G_{0}=\mathscr{A}\left[p^{n}\right]
$$

by closed flat subgroup schemes such that successive subquotients $G_{i} / G_{i+1}$ have order $p$.

THEOREM 3.5. Let $K=\mathbf{Q}(\sqrt{m})$ be an imaginary quadratic field with class number one and let $p$ be an odd prime number such that $p$ does not ramify in $K$ and $(m / p)=1$. Suppose that $p$ does not divide the class number of $K\left(\zeta_{p}\right)$. Let $A$ be an abelian variety over $K$ with bad reduction only at the primes of $K$ over $p$. Then $A$ has no complex multiplication over $K$.

Proof. Assume $A$ has complex multiplication over $K$. Since the class number of $K$ is equal to 1 , any extension over $\mathcal{O}_{K}$ of constant $p$-group schemes by one another is constant. Considering the Cartier dual, any extension over $\mathcal{O}_{K}$ of diagonalizable $p$-group schemes by one another is diagonalizable. Note that the only group schemes over $\mathcal{O}_{K}$ of order $p$ are $\mu_{p}$ and $\mathbf{Z} / p \mathbf{Z}$ by Proposition 3.3. By Lemma 3.4 and the proof of [9, Theorem 2.1], there is an exact sequence

$$
0 \rightarrow M \rightarrow \mathscr{A}\left[p^{n}\right] \rightarrow C \rightarrow 0
$$

with $M$ diagonalizable and $C$ constant. By the proof of $[9$, Theorem 2.1], we have that $\operatorname{dim} A=0$.

Let $K=\mathbf{Q}(\sqrt{-19})$ and set $p=5$. Since the class number of the cyclotomic field $\mathbf{Q}\left(\zeta_{95}\right)$ is not divisible by $p$ (see [13]), it follows that the class number of $K\left(\zeta_{p}\right)$ is not divisible by $p$ by Lemma 3.2. As a corollary, we get the following.

THEOREM 3.6. There do not exist non-zero abelian varieties over $K=$ $\mathbf{Q}(\sqrt{-19})$ with good reduction everywhere and complex multiplication over $K$.

\subsection{Elliptic curves over certain number fields with good reduction}

It is well known that there is no elliptic curves over $\mathbf{Q}$ with good reduction. On the other hand, several examples of such curves over quadratic fields are known. An elliptic curve defined over a number field $k$ is called $g$-admissible if it satisfies the conditions below: 
1. it has good reduction over $k$,

2. it has a $k$-rational point of order 2 ,

3. it admits a global minimal model.

If it satisfies only (1) and (2), then it is called admissible. In his paper [2], Comalada showed that there exists an admissible elliptic curve over $K=\mathbf{Q}(\sqrt{m})$ $(0<m<100)$ if and only if

$$
m=6,7,14,22,38,41,65,77,86 \text {. }
$$

Example. The elliptic curve $E$ over $K=\mathbf{Q}(\sqrt{6})$ with Weierstrass equation

$$
y^{2}+\sqrt{6} x y-y=x^{3}-(2+\sqrt{6}) x^{2}
$$

is $g$-admissible. This can be seen from the fact that the discriminant of $E$ is equal to the unit $(5+2 \sqrt{6})^{3}$. The three points of order 2 of $E$ have their $x$-coodinates equal to $x=-\frac{1}{2}$ and $\frac{1+\sqrt{6} \pm(\sqrt{-2}+\sqrt{-3}) i}{2}$ respectively. Their $y$-coordinates are given by $y=\frac{-\sqrt{6} x+1}{2}$. The point with $x=-\frac{1}{2}$ is the only 2-rational point that is rational over $K$. The curve $E$ has exactly six points defined over $K$. They are $(0,0)$ and its multiples $(2+\sqrt{6},-5-2 \sqrt{6}), \quad\left(-\frac{1}{2}, \frac{1}{2(\sqrt{6}-2)}\right)$,
$(2+\sqrt{6}, 0),(0,1)$ and $\infty$.

Let $K$ be a number field and let $p$ be an odd prime number. Let $E$ be an elliptic curve over $K$ with good reduction. We now consider the $K$-rational points of order $p$ in the elliptic curve $E$. Suppose there exists a $K$-rational point $P$ of order $p$ in the elliptic curve $E$. Using the Weil pairing $e_{p}: E[p] \times E[p] \rightarrow$ $\mu_{p}$, we can define a map $E[p] \rightarrow \mu_{p}$ by $Q \mapsto e_{p}(P, Q)$. Since the point $P$ is rational over $K$, this map gives an exact sequence

$$
0 \rightarrow \mathbf{Z} / p \mathbf{Z} \rightarrow E[p] \rightarrow \mu_{p} \rightarrow 0 .
$$

of $\operatorname{Gal}(\bar{K} / K)$-modules. Let $\mathscr{E}$ be the Néron model of the elliptic curve $E$ over $\mathcal{O}_{K}$. Since the elliptic curve $E$ has good reduction over $K$, note that $\mathscr{E}[p]$ is a finite flat group scheme over $\mathcal{O}_{K}$.

Lemma 3.7. Suppose that the ramification index $e_{\mathfrak{p}}$ satisfies $e_{\mathfrak{p}}<p-1$ for all primes $\mathfrak{p}$ of $K$ over $p$. Then the exact sequence (3) of $\mathrm{Gal}(\bar{K} / K)$-modules induces an exact sequence

$$
0 \rightarrow \mathbf{Z} / p \mathbf{Z} \rightarrow \mathscr{E}[p] \rightarrow \mu_{p} \rightarrow 0
$$

of finite flat group schemes over $\mathcal{O}_{K}$.

Proof. For any finite flat group schemes $G$ over $\mathcal{O}_{K}$, there is a one-to-one correspondence between closed flat subgroup schemes between $G$ over $\mathcal{O}_{K}$ and $G \otimes_{\mathcal{O}_{K}} K$ over $K$. For any finite flat group schemes $G$ over $\mathcal{O}_{K, \mathfrak{p}}$ of $p$-power order, by the assumption, $G$ is uniquely determined up to isomorphism by the 
isomorphism type of its generic fiber (see [12, Propositon 4.5.1]). Therefore a constant group scheme $\mathbf{Z} / p \mathbf{Z}$ is a subgroup scheme of $\mathscr{E}[p]$ over $\mathcal{O}_{K}$. There exists an exact sequence

$$
0 \rightarrow \mathbf{Z} / p \mathbf{Z} \rightarrow \mathscr{E}[p] \rightarrow G \rightarrow 0
$$

of finite flat group schemes over $\mathcal{O}_{K}$. Since $G \otimes K$ is isomorphic to a diagonalizable group scheme $\mu_{p}$ by the exact sequence (3), $G$ is isomorphic to a diagonalizable group scheme $\mu_{p}$ over $\mathcal{O}_{K}$. This completes the proof.

Combining the above result with Theorem 2.2, we get the following result:

THEOREM 3.8. Let $K$ be a number field having a real place and let $p$ be a prime number. Suppose that $p$ does not divide the class number of $K\left(\zeta_{p}\right)$ and the ramification index $e_{\mathfrak{p}}$ satisfies $e_{\mathfrak{p}}<p-1$ for all primes $\mathfrak{p}$ of $K$ above $p$. Let $E$ be an elliptic curve over $K$ with good reduction. Then $E$ has no $K$-rational points of order $p$.

Proof. Suppose there exists a $K$-rational point of order $p$ in the elliptic curve $E$. By Lemma 3.7, there exists an exact sequence

$$
0 \rightarrow \mathbf{Z} / p \mathbf{Z} \rightarrow \mathscr{E}[p] \rightarrow \mu_{p} \rightarrow 0
$$

of finite flat group schemes over $\mathcal{O}_{K}$. Set $E=E_{1}$. Since the above exact sequence of finite flat group schemes over $\mathcal{O}_{K}$ is split by Theorem 2.2, there exists an elliptic curve $E_{2}$ over $K$ and a $K$-isogeny $E_{1} \rightarrow E_{2}$ with kernel $\mu_{p}$. Then the image of the Galois submodule $\mathbf{Z} / p \mathbf{Z}$ gives a point of order $p$ in $E_{2}$. Continuing in this fashion, we obtain a sequence of $K$-isogenies

$$
E_{1} \rightarrow E_{2} \rightarrow \cdots,
$$

where each isogeny has kernel $\mu_{p}$. Since all the $E_{i}$ has good reduction over $K$, we see that $E_{i} \simeq E_{j}$ for some $i<j$ (see [4, Satz 6]). Composing our $K$-isogenies gives a endomorphism $f: E_{i} \rightarrow E_{i}$ defined over $K$. If $P_{i} \in E_{i}(K)$ is the image of $P \in E(K)$, then by construction $P_{i} \notin \operatorname{Ker} f$. Since $\operatorname{deg} f$ is a power of $p$, we see that $f$ is a non-scalar endomorphism. Therefore the elliptic curve $E_{i}$ has complex multiplication by some order $\mathcal{O}$ in an imaginary quadratic field $K^{\prime}$, and we have an isomorphism (see [11, Ch. 2, Proposition 1.1])

$$
[\cdot]: \mathcal{O} \simeq \operatorname{End}\left(E_{i}\right)
$$

such that for any invariant differential $\omega \in \Omega_{E_{i}}$ on $E_{i}$,

$$
[\alpha]^{*} \omega=\alpha \omega \text { for all } \alpha \in \mathcal{O} \text {. }
$$

Let $\alpha$ be the element of $\mathcal{O}$ such that $[\alpha]=f \in \operatorname{End}_{K}\left(E_{i}\right)$. Considering the action of $\operatorname{End}_{K}\left(E_{i}\right)$ on $\mathrm{H}^{0}\left(E_{i} / K, \Omega_{E_{i}}\right) \simeq K$, we have $\alpha \in K \cap K^{\prime}=\mathbf{Q}$. This is a contradiction.

As a corollary, we get the following result: 
COROllary 3.9. Let $K=\mathbf{Q}(\sqrt{6})$ or $\mathbf{Q}(\sqrt{7})$. Let $E$ be an elliptic curve over $K$ with good reduction. Then $E$ has no $K$-rational points of order $p$ for any prime number $p \geq 5$.

Proof. Let $\mathscr{E}$ be the Néron model of $E$ over $\mathcal{O}_{K}$. Since $E$ has good reduction at the prime of $K$ over 2, the elliptic curve $\mathscr{E}\left(\mathbf{F}_{2}\right)$ has at most $3+2 \sqrt{2}<7$ points. Therefore $E$ has no $K$-rational points of order $p$ for any prime number $p \geq 7$. Set $p=5$. We consider the case $K=\mathbf{Q}(\sqrt{6})$. Since the class number of the cyclotomic field $\mathbf{Q}\left(\zeta_{120}\right)$ is not divisible by $p$ (see [13]), the class number of $K\left(\zeta_{p}\right)$ is not divisible by $p$ by Lemma 3.2. By Theorem 3.8, the elliptic curve $E$ has no $K$-rational points of order $p$. We now consider the case $K=\mathbf{Q}(\sqrt{7})$. Since the class number of the cyclotomic field $\mathbf{Q}\left(\zeta_{140}\right)$ is not divisible by $p$ (see [13]), the class number of $K\left(\zeta_{p}\right)$ is not divisible by $p$ by Lemma 3.2. This completes the proof by Theorem 3.8.

The following table is all taken from [2] and [7]. In the table, all the isomorphism classes of $g$-admissible elliptic curves having good reduction over the three fields $K=\mathbf{Q}(\sqrt{m})(m=6,7,14)$ are listed. Each isomorphism class contains a curve having a Weierstrass equation of the form

$$
y^{2}=x^{3}+a_{2} x^{2}+a_{4} x
$$

on which the point $(0,0)$ is of order 2 . For each curve, the data given in the table are Comalada's code $E_{i}, m, a_{2}, a_{4}$, the $j$-invariant, and the torsion subgroup $T$ of the Mordell-Weil group. The coefficients $a_{2}, a_{4}$ and the $j$-invariant are given by expressions containing the fundamental unit $\varepsilon$ of $K$ and its conjugate $\bar{\varepsilon}$.

Table 1. Elliptic curves having good reduction over $\mathbf{Q}(\sqrt{6}), \mathbf{Q}(\sqrt{7}), \mathbf{Q}(\sqrt{14})$

\begin{tabular}{|c|c|c|c|c|c|}
\hline & $m$ & $a_{2}$ & $a_{4}$ & $j$ & $T$ \\
\hline$E_{1}$ & 6 & $-2(\varepsilon-1)$ & $4 \varepsilon$ & $(20)^{3}$ & $\mathbf{Z} / 6 \mathbf{Z}$ \\
\hline$E_{3}$ & 6 & $-14(\varepsilon-1)$ & $4 \bar{\varepsilon}$ & $64\left(4 \varepsilon^{4}+1\right)^{3} / \varepsilon^{4}$ & $\mathbf{Z} / 2 \mathbf{Z}$ \\
\hline$E_{5}$ & 6 & $14(\varepsilon-1) \varepsilon$ & $4 \varepsilon$ & $64\left(4 \varepsilon^{4}+1\right)^{3} / \varepsilon^{4}$ & $\mathbf{Z} / 6 \mathbf{Z}$ \\
\hline$E_{7}$ & 7 & $-\left(1+2 \varepsilon^{2}\right)$ & $16 \varepsilon^{3}$ & $(255)^{3}$ & $\mathbf{Z} / 2 \mathbf{Z} \times \mathbf{Z} / 2 \mathbf{Z}$ \\
\hline$E_{9}$ & 7 & $2\left(1+2 \varepsilon^{2}\right)$ & 1 & $\left(256 \varepsilon^{2}+\bar{\varepsilon}\right)^{3}$ & $\mathbf{Z} / 4 \mathbf{Z}$ \\
\hline$E_{11}$ & 7 & $-2\left(1+2 \varepsilon^{2}\right)$ & 1 & $\left(256 \varepsilon^{2}+\bar{\varepsilon}\right)^{3}$ & $\mathbf{Z} / 2 \mathbf{Z}$ \\
\hline$E_{13}$ & 7 & $8 \varepsilon-1$ & $16 \varepsilon^{2}$ & $(-15)^{3}$ & $\mathbf{Z} / 4 \mathbf{Z}$ \\
\hline$E_{14}$ & 7 & $-(8 \varepsilon-1)$ & $16 \varepsilon^{2}$ & $(-15)^{3}$ & $\mathbf{Z} / 4 \mathbf{Z}$ \\
\hline$E_{15}$ & 14 & $-3(\varepsilon-1) / 2$ & $16 \varepsilon$ & $(-15)^{3}$ & $\mathbf{Z} / 2 \mathbf{Z}$ \\
\hline$E_{17}$ & 14 & $3(\varepsilon-1)$ & $-\varepsilon$ & $(255)^{3}$ & $\mathbf{Z} / 2 \mathbf{Z}$ \\
\hline
\end{tabular}


Acknowledgement. I would like to thank Professor Toshiyuki Katura, who gave me various advice and useful comments.

\section{REFERENCES}

[ 1] M. Artin, Algebraization of formal moduli, II, Existence of modifications, Ann. of Math. 91 (1970), 88-135.

[2] S. Comalada, Elliptic curves with trivial conductor over quadratic fields, Pacific J. Math. 144 (1990), 237-258.

[3] B. Conrad, Finite group schemes over base with low ramification, Compos. Math. 119 (1999), 239-320.

[ 4 ] G. Faltings, Endlichkeitssätze für abelsche Varietäten über Zahlkörpern, Invent. Math. 83 (1983), 349-366.

[5] J.-M. Fontaine, Groupes p-divisible sur les corps locaux, Astérisque (1977), 47-48.

[6] N. Katz And B. Mazur, Arithmetic moduli of elliptic curves, Ann. of Math. Stud. 108, Princeton University Press, Princeton, 1985.

[ 7] M. KIDA, Reduction of elliptic curves over certain real quadratic number fields, Math. Comp. 68 (1999) 228, 1679-1685.

[ 8 ] F. Oort and J. Tate, Group schemes of prime order, Ann. Sci. École Norm. Sup. 3 (1970), $1-21$.

[9] R. Schoof, Abelian varieties over cyclotomic fields with good reduction, Math. Ann. 325 (2003), 413-448.

[10] J.-P. Serre and J. Tate, Good reduction of abelian varieties, Ann. of Math. 88 (1968), 492-517.

[11] J. H. Silvermann, Advanced topics in the arithmetic of elliptic curves, Graduate texts in math. 151, Springer-Verlag, Berlin-Heidelberg-New York, 1994.

[12] J. TATE, Finite flat group schemes, Modular forms and Fermat's last theorem, SpringerVerlag, Berlin-Heidelberg-New York, 1997, 121-154.

[13] L. C. Washington, Introduction to cyclotomic fields, Graduate texts in math. 83, SpringerVerlag, Berlin-Heidelberg-New York, 1982.

Masaya Yasuda

FUJITSU LABORATORIES LTD.

1-1, KamikodanaKa 4-CHOME

NAKAHARA-KU, KAWASAKI 211-8588

JAPAN

E-mail: myasuda@labs.fujitsu.com 